\title{
«Clinically silent» somatotropinoma
}

\author{
Lipatenkova A.K., Dzeranova L.K., Pigarova E.A.,
}

Endocrinology Research Centre, Moscow, Russia

\section{Introduction}

Somatotropinomas are typically recognized when $\mathrm{GH}$ excessively secretion causes acromegaly. "Silent» somatotroph adenomas (immunohistochemical evidence of $\mathrm{GH}$ excess without biochemical or clinical evidence) and "clinically silent» somatotroph adenomas (immunohistochemical and biochemical evidence without clinical evidence) is unknown.

\section{The aim}

to describe the clinical course of a patient with «clinically silent» somatotroph adenoma.

\section{Case presentation}

In a female patient disease presented at the age of 22 years with oligomenorrhea and hyperprolactinemia - PRL $881.37 \mathrm{mU} / \mathrm{l}$ (127637) for wich she received cabergoline $0.25 \mathrm{mg}$ per week with induction of normoprolactinemia and normalization of menstrual cycle. Within a year MRI revealed endo-suprasellar pituitary adenoma $16 * 15^{*} 13 \mathrm{~mm}$, without chiasm compression. The levels of TSH, cortisol, LH, FSH, prolactin were in normal ranges. These changes were regarded as nonfunctioning pituitary adenoma and annual follow-up was recommended.

Subsequent MRIs showed an enlargement $\left(21^{*} 18^{* 17} \mathrm{~mm}\right)$, mainly due to suprasellar component without chiasm involvement (pic.1) ACTH, cortisol, PRL, LH, FSH were within reference ranges and a decrease of free T4 $8.1 \mathrm{pmol} / \mathrm{l}$ (9-20) and IGF-1 elevation $816.9 \mathrm{ng} / \mathrm{ml}$ (128-315) were noted. The high fasting $\mathrm{GH}$ levels confirmed biochemical acromegaly (9.1-6.3-11.3-11.1-13.3 $\mathrm{ng} / \mathrm{ml}$ ) as at physical examination there were no clinical signs of this disease. Patient got levothyroxine $25 \mathrm{mg}$ a day to correct secondary hypothyroidism.

\section{Picture 1. MRI before surgical treatment}

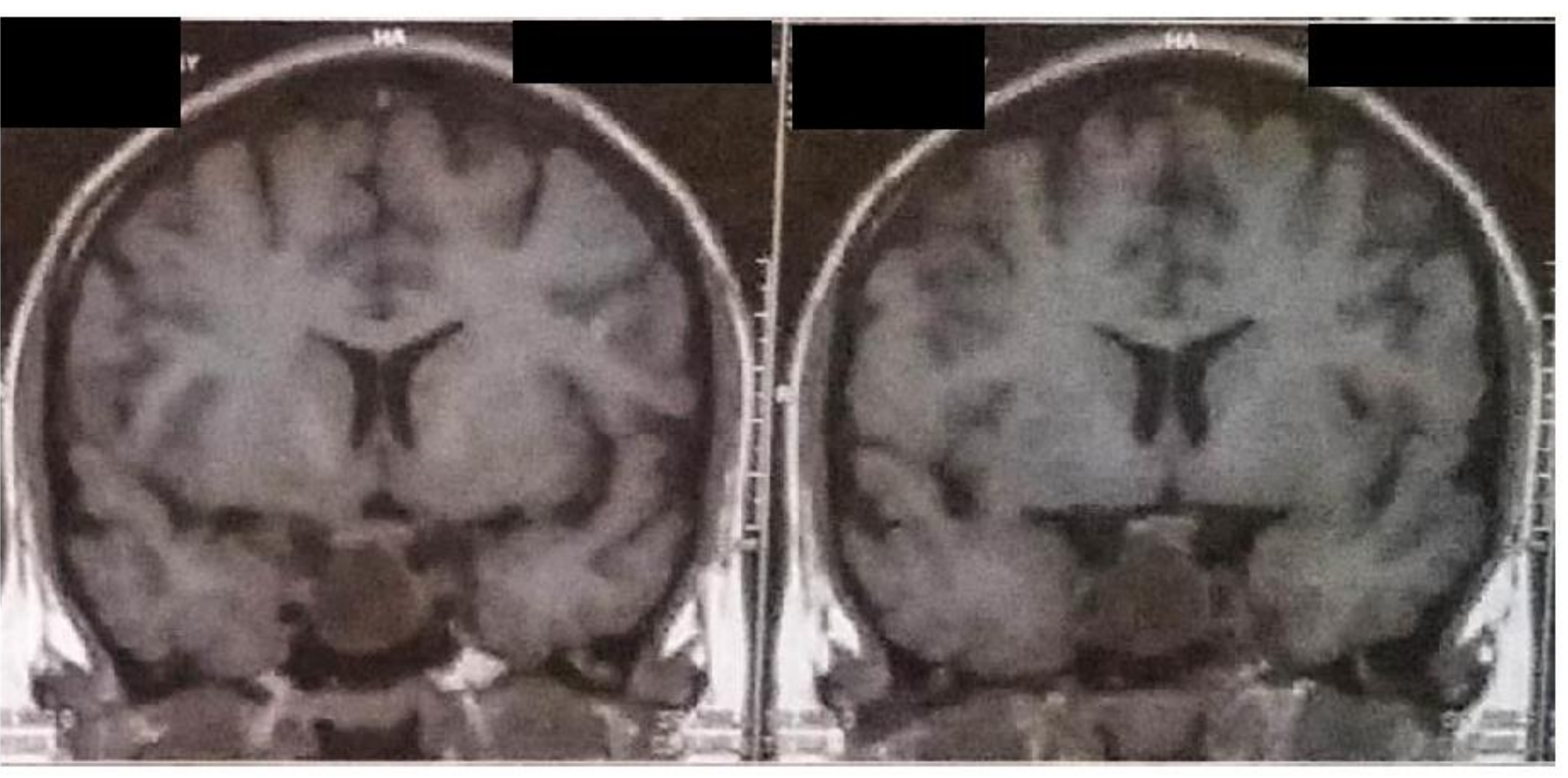

Transsphenoidal surgery was performed. Immunohistochemical staining showed that tumor cells were strongly reactive to $\mathrm{GH}$ and relatively mildly reactive to PRL, with ki-67 2\% confirming the presence of clinically «silent» somatotropinoma (pic.2).

After 3, 6, 12 months after the surgery the levels of IGF1 remain in normal ranges: $231 \mathrm{ng} / \mathrm{ml}(60-280)-215-$ 228. The brain MRI was repeated after 12 months thereafter with no signs of adenoma.

At the moment patient has a normal menstrual? Prolactin remains in reference ranges. L-thyroxine was canceled and free T4 in the dynamics of 3 and 6 months after removal was stable 13-16.2 pmol/l.

\section{Picture 2. Immunohistochemical staining for $\mathrm{Ki}-67$ and $\mathrm{GH}$}

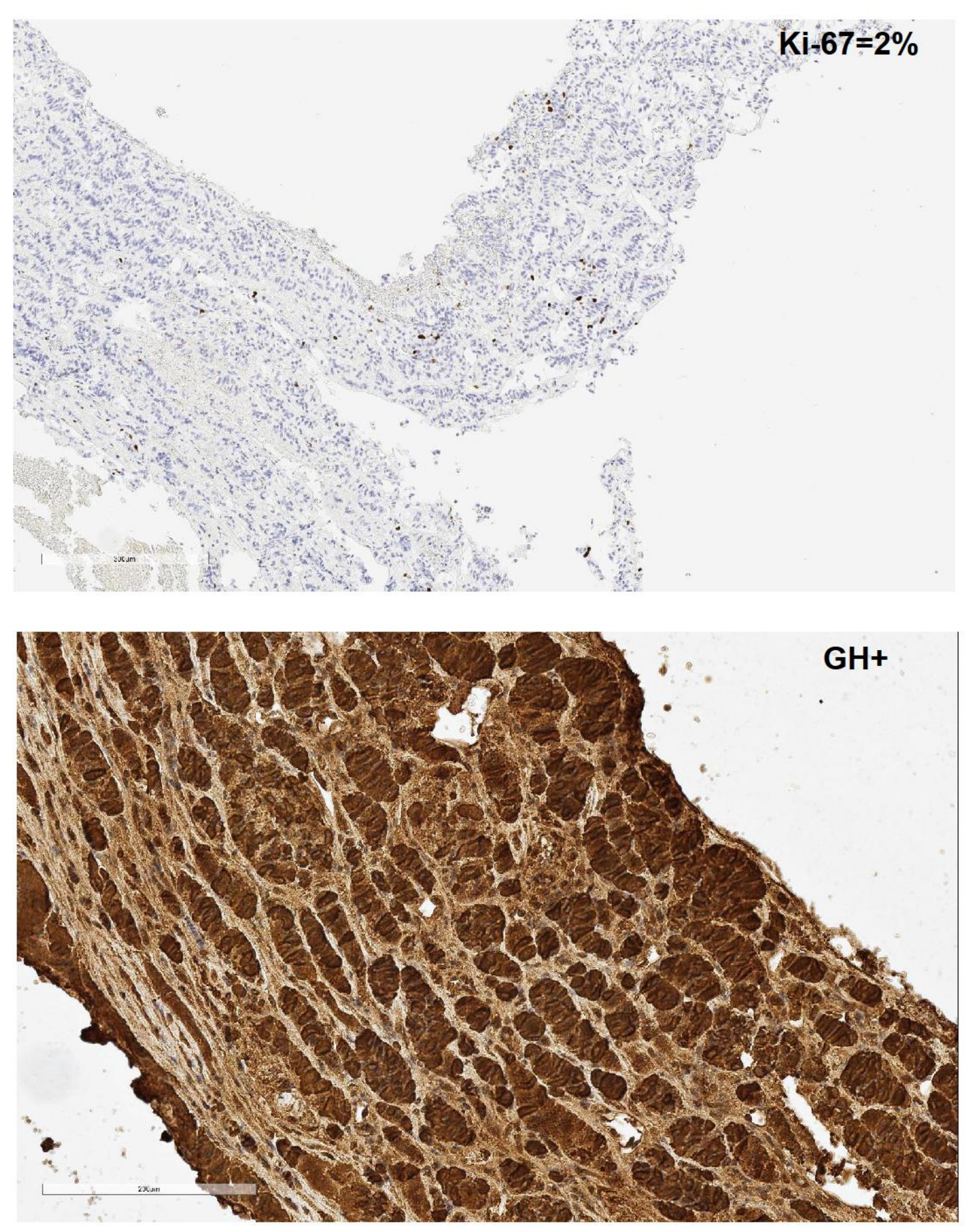

\section{Conclusions:}

Clinically silent somatotroph adenomas with biochemical and immunohistochemical evidence of growth hormone excess but absence of clinical features of acromegaly were first described nearly 30 years ago. There were no significant biochemical or histological differences between clinically active and clinically silent somatotroph adenomas. This case demonstrates the need to perform an extensive hormonal testing for all patients with presumably non-active pituitary adenoma. 